\title{
Zolpidem, a clinical hypnotic that affects electronic transfer, alters synaptic activity through potential GABA receptors in the nervous system without significant free radical generation
}

\author{
Peter Kovacic ${ }^{1, *}$ and Ratnasamy Somanathan ${ }^{1,2}$ \\ 'Department of Chemistry; San Diego State University; San Diego, California USA; ${ }^{2}$ Centro de Graduados e Investigación del Instituto Tecnológico de Tijuana; Tijuana, B.C. \\ Mexico.
}

\begin{abstract}
Abbreviations: ET, electron transfer; SAR, structure activity relationship; CNS, central nervous system; ROS, reactive oxygen species; OS, oxidative stress; MEP, molecular electrostatic potential; GABA, gamma-amino butyric acid; Arg, arginine; Glu, glutamic acid; EEG, electroencephalograph; GSH, glutathione; FAA, flavone-8-acetic acid; DMXAA, 5,6-dimethylxanthenone-4-acetic acid; BZ, benzodiazepine; AA, amino acid
\end{abstract}

Key words: zolpidem, electrochemistry, receptors, cell signaling, SAR, neuroscience

Zolpidem (trade name Ambien) has attracted much interest as a sleep-inducing agent and also in research. Attention has been centered mainly on receptor binding and electrochemistry in the central nervous system which are briefly addressed herein. A novel integrated approach to mode of action is presented. The pathways to be discussed involve basicity, reduction potential, electrostatics, cell signaling, GABA receptor binding, electron transfer (ET), pharmacodynamics, structure activity relationships (SAR) and side effects. The highly conjugated pyridinium salt formed by protonation of the amidine moiety is proposed to be the active form acting as an ET agent. Extrapolation of reduction potentials for related compounds supports the premise that zolpidem may act as an ET species in vivo. From recent literature reports, electrostatics is believed to play a significant role in drug action.

The pyridinium cation displays molecular electrostatic potential which may well play a role energetically or as a bridging mechanism. An SAR analysis points to analogy with other physiologically active xenobiotics, namely benzodiazepines and paraquat in the conjugated iminium category. Inactivity of metabolites indicates that the parent is the active form of zolpidem. Absence of reactive oxygen species and oxidative stress is in line with minor side effects. In contrast, generally, the prior literature contains essentially no discussion of these fundamental biochemical relationships. Pharmacodynamics may play an important role. Concerning behavior at the blood-brain barrier, useful insight can be gained from investigations of the related cationic anesthetics that are structurally related to acetyl choline. Evidently, the neutral form

\footnotetext{
*Correspondence to: Peter Kovacic; Department of Chemistry; San Diego State University; San Diego, California 92182-1030 USA; Email: pkovacic@sundown. sdsu.edu

Submitted: 12/19/08; Revised: 01/06/09; Accepted: 01/16/09

Previously published online as an Oxidative Medicine and Cellular Longevity E-publication: http://www.landesbioscience.com/journals/oximed/article/7859
}

of the drug penetrates the neuronal membrane, with the salt form operating at the receptor. The pathways of zolpidem have several clinical implications since the agent affects sedation, electroencephalographic activity, oxidative metabolites and receptors in the central nervous system. The drug acts at the GABA(A) receptor benzodiazepine site, displaying high and intermediate affinities to various receptor regions. Structural features for tight binding were determined. The sedative and anticonvulsant activities are due to its action on the alpha-1-GABA(A) receptors. One of the common adverse responses to zolpidem is hallucinations. Proposed mechanisms comprise changes in the $\operatorname{GABA}(\mathrm{A})$ receptor, pharmacodynamic interactions involving serotonin and neuronal-weak photon emission processes entailing redox phenomena. Reports cite cases of abuse with cravings based on anxiolytic and stimulating actions. It is important to recognize that insight concerning processes at the fundamental, molecular level can translate into beneficial results involving both positive and adverse side effects. In order for this to occur, interdisciplinary interaction is necessary. Suggestions are made for future research aimed at testing the various hypotheses.

\section{Introduction}

Zolpidem (Ambien) (Fig. 1) (tartrate salt) has attracted considerable recent attention in connection with its widespread use as a preferential hypnotic with minor side effects. There has been the usual focus on receptor binding. In addition, since the brain is the major organ involved, many investigations have been devoted to electrochemical action involving the central nervous system (CNS). However, as with most other drugs, there is negligible focus on events at the fundamental level involving the translation of binding into physiological action. The present report provides an integrated approach consisting of drug basicity, receptor docking, electron transfer (ET), electrochemistry including reduction potential, electrostatics and the CNS, cell signaling, pharmacodynamics, structure 
activity relationships (SAR), side effects, medical implications and future research for hypothetical testing.

The preponderance of bioactive substances or their metabolites incorporate ET functionalities, which, we believe, play an important role in physiological responses. The main groups include quinones (or phenolic precursors), metal complexes (or complexors), aromatic nitro compounds (or reduced nitroso or hydroxylamine derivatives) and conjugated iminiums (or imines). The focus of the present proposal is the iminium category. There are two principal pathways that can result from ET, one being redox cycling with generation of reactive oxygen species (ROS) and oxidative stress (OS). We are concerned with the other route in which ET involves interaction with the CNS, resulting in this case in sedative effects. Drugs in other categories are cited for which there is also ET with little or no participation of ROS and OS.

Electron transfer is probably the most prevalent and important process in chemical transformation. ${ }^{1}$ The generality and unifying aspects are demonstrated by involvement in all areas of the physical and biological sciences. The term bioelectronome is applied to participation in the life sciences. Examples are receptor chemistry ${ }^{1}$ and cell-signaling mechanism. ${ }^{2}$ Extensive evidence is documented supporting mode of action for anti-infective drugs, ${ }^{3}$ anticancer agents, ${ }^{4}$ carcinogens ${ }^{5}$ and major organ toxins. ${ }^{6-15}$

Since electrochemistry plays an important role in biofunctioning, including the CNS, more attention should be devoted to this area, particularly fundamental aspects. A neglected topic is determination of reduction potential which provides information concerning the feasibility of ET by exogenous agents. If the reduction potential is more positive than $-0.5 \mathrm{~V}$, then ET is a possibility in vivo. Requisite electron donors reside mainly in protein side chains in the form of disulfide, phenolic oxygen or conjugated amine species.

In the electrochemical category, studies demonstrate a role for electrostatic forces in a variety of areas. The term bioelectrostatics is a label used in the biochemical area in which widespread participation is documented. ${ }^{16}$ Energetics appears to play a significant role. Subjects addressed are enzymes, membranes, chromosomes, histamine, receptors, the Hofmeister effect, plant chemistry and evolutionary development. A recent hypothesis proposes that electrostatic force is a factor in receptor-ligand action, based on the molecular electrostatic potential (MEP) associated with ions and dipoles. ${ }^{17}$ Energetics and bridging may be important actors. Application is also made to phosphate and sulfate anions in cell signaling, ${ }^{18}$ as well as to metal cations, such as $\mathrm{Ca}, \mathrm{Mg}, \mathrm{Zn}, \mathrm{Fe}$ and $\mathrm{Cu}^{19}{ }^{19}$ There is appreciable research dealing with MEP in relation to DNA chemistry. ${ }^{20}$ Various other aspects of electrochemistry are addressed elsewhere. ${ }^{11}$

It should be emphasized that physiological activity of endogenous and exogenous agents is often complex and multifaceted. Knowledge of events at the basic molecular level can result in practical application to medicine.

\section{Basicity}

The amidine structure possesses enhanced basicity due to resonance stabilization of the protonated cation. Studies show that alkyl substituents enhance $\mathrm{pKa}(-12)$, whereas the phenyl group decreases the value to about $8 .^{21}$ The unsaturated vinyl group should also act to decrease. Another factor comes into play in connection with the amidine present in Figure 1. Protonation yields a resonance-stabilized

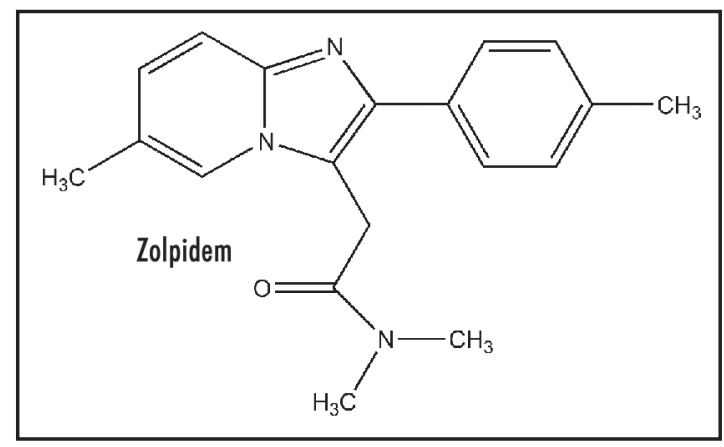

Figure 1. Zolpidem.

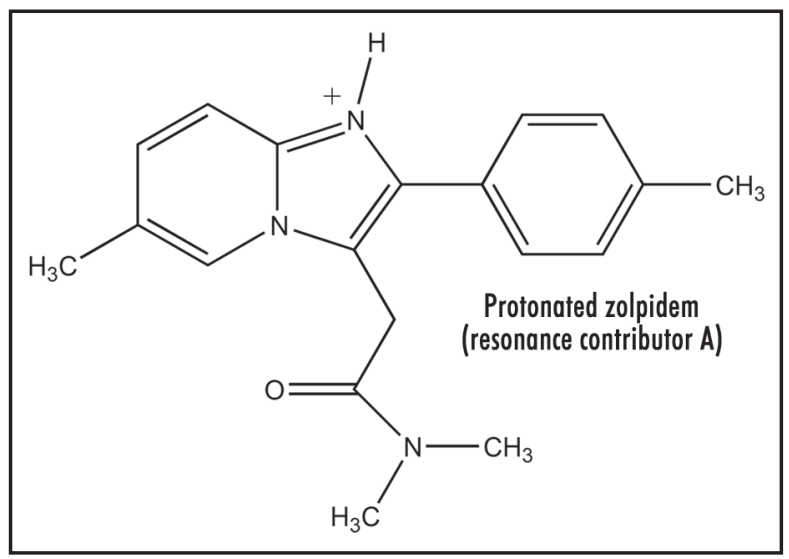

Figure 2. Protonated zolpidem (resonance contributor A).

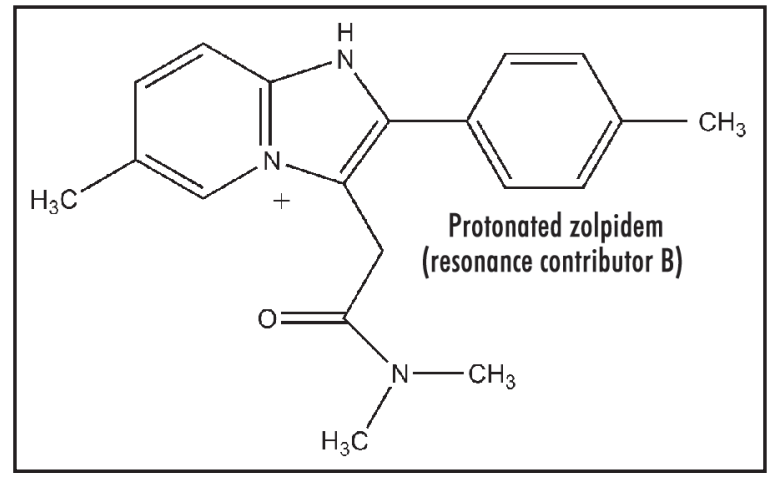

Figure 3. Protonated zolpidem (resonance contributor B).

cation incorporating the contributors Figures 2 and 3. Form Figure 3 is the major one as a result of resonance stabilization by the aromatic pyridinium ion, which also should be a driving force for protonation. Presence of the highly conjugated pyridinium salt apparently has electrochemical consequences (see below). The importance of these structural features seems not to have been considered previously.

\section{Reduction Potential}

Except for redox enzymes, ease of electron uptake has attracted little attention, e.g., in the case of therapeutic drugs, even though it is an important property. Revealing electrochemical studies are reported for aromatic hydrocarbon analogs of Figure 3 that provide mechanistic 


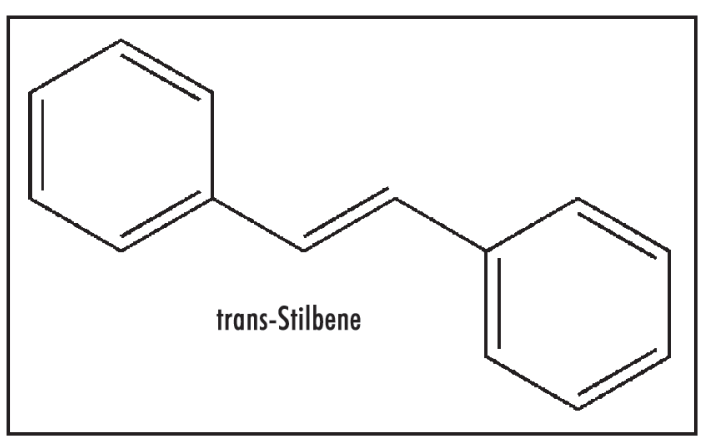

Figure 4. trans-Stilbene.

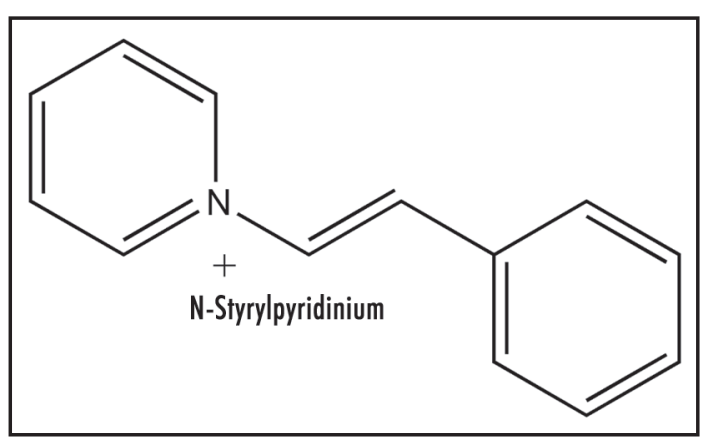

Figure 5. N-Styrylpyridinium.

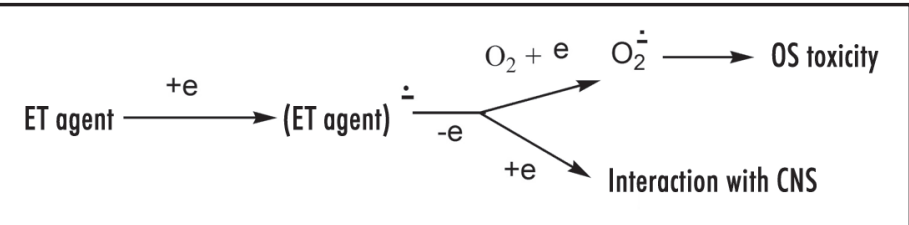

Scheme 1. Mechanism of ET action.

insight. Biphenyl possesses a reduction potential of $-2.05 \mathrm{~V}$, and as expected, trans-stilbene (Fig. 4) has a more positive value of -1.64 $\mathrm{V}^{22}$ Thus, the vinyl link provides appreciable enhancement of about $0.4 \mathrm{~V}$. A report is available for relevant data on $\mathrm{N}$-phenylpyridinium, a simple model for Figure 3, containing a figure of $-0.84 \mathrm{~V}^{23}$ The structure in Figure 3 incorporates a trans-pyridinium-benzene-vinyl arrangement (Fig. 5) quite similar to that of trans-stilbene. Hence, extending the conjugation in $\mathrm{N}$-phenylpyridinium by insertion of a vinyl moiety to form the N-styrylpyridinium (Fig. 5) should positively increase the reduction potential by about $0.4 \mathrm{~V}$. The bicyclic moiety results in enhancement due to restricted rotation. The aromatic methyl group has a very small negative effect. ${ }^{22}$ As a result, the estimated reduction potential for Figure 3 would be about $-0.4 \mathrm{~V}$, which is within the range permitting ET in vivo. Positive charge in Figure 3 would facilitate ease of electron uptake. Whether reduction potential is quite positive or less so may be a factor in whether electron loss from the ET functionality occurs to oxygen or by another route. Scheme 1 provides more detail on the mode of action by zolpidem involving ET. This ET property might well play a role in the beneficial effects of zolpidem, as well as the undesirable side reactions (see below).

A related substance, 1,1'-dimethyl-4,4'-bipyridinium (paraquat), is able to participate in ET and redox cycling with formation of ROS, due to its favorable reduction potential. ${ }^{6}$ The herbicide, like Figure 3, contains a highly conjugated iminium-like structure, making it part of one of the important ET classes.

\section{Pharmacodynamics}

This portion deals with the drug state, whether ionic or uncharged, in relation to migration and binding. Concerning behavior at the blood-brain barrier, useful insight can be gained from investigations of the related cationic anesthetics that are structurally similar to acetyl choline. ${ }^{24}$ The majority of these anesthetics have $\mathrm{pKa}$ values such that when aqueous solutions of their salts, e.g., hydrochlorides, are injected into the extracellular fluid, they will equilibrate so that the neutral form will readily penetrate through the neuronal membrane. Once inside, this form would again equilibrate to form the ionic, active species, which presumably can then interact with the putative receptor. Hence, a similar scenario may pertain to zolpidem base and the cation form. At the receptor site, attachment might occur in several ways. In one case, the charged form is involved by comparison with other polar entities, such as acetylcholine. In the other situation, the base is protonated at the binding domain, in keeping with the strong nature of the attachment. The requisite proton might be provided by unionized carboxyl of acidic amino acids or the acidic hydrogen of protonated amino acids. It may be relevant that a hydrogen bond with arginine, presumably in guanidinium form, was found involving a particular conformation of zolpidem in the GABA(A) receptor. ${ }^{25}$ In another report, Arg and Glu were designated important features for docking. ${ }^{26}$ Hence, in addition to zolpidem interaction with the guanidinium proton, the carboxyl of Glu might also be a source for salt formation.

Since the drug in ionic form is acting catalytically in ET, only a small amount is needed. Receptor site binding is usually not covalent, allowing reversibility and escape of the ligand. Thus, the drug is eventually oxidized to inactive products (see metabolites) resulting in cessation of activity.

\section{Electrostatics}

This unifying theme, discussed in the Introduction, might also apply to zolpidem. In salt form the pyridinium cation possesses a strong molecular electrostatic potential which could influence drug action and side effects. There is prior mechanistic discussion of electrostatics in connection with various physiologically active $\mathrm{N}$-containing organic cations, such as acetyl choline and zwitterions of GABA and $\alpha$-amino acids. ${ }^{17}$ In a critical analysis of the Hofmeister series dealing with various effects of ions, the most active was the ammonium type, and the most active anions were sulfate and phosphate. ${ }^{16}$ The association with involvement of these ions in cell signaling was pointed out. An investigation led to the conclusion that electrostatic forces play a significant role in the Hofmeister series. ${ }^{27}$

\section{Electrochemistry in the CNS}

Extensive literature exists in this category, part of which deals with electroencephalograph (EEG) activity. Zolpidem increases EEG beta frequencies, characterstic for BZs. ${ }^{28}$ The drug seems to amplify the relation between behavior and the EEG. A study of dosedependent EEG effects of zolpidem provides evidence for GABA(A) receptor subtype selectivity in vivo. ${ }^{29} \mathrm{EEG}$ in relation to sleep was examined. The influences on sleep EEG are primarily mediated by 
alpha-1 GABA(A) receptors, and are distinct from changes induced by BZs. ${ }^{30}$ An EEG investigation was made in connection with sleep deprivation. ${ }^{31}$ After administration of zolpidem, power in certain $\mathrm{HZ}$ ranges and bands was reduced. The drug and sleep deprivation have different effects on the EEG power spectra. The sedative, unlike triazolam and zopiclone, had a much milder reducing effect on the frequency of hippocampal theta activity and suppressing effect on REM sleep. ${ }^{32}$ Various studies were carried out on the effects on CNS currents. ${ }^{33-41}$ A reduction of reticulate neuronal activity by the sedative was noted. ${ }^{42}$ Cerebellar Purkinje neurons are sensitive to enhancement of GABA by zolpidem. ${ }^{43}$

The drug markedly diminished the levels of cerebral cGMP in the rat. ${ }^{44}$ Findings are consistent with the hypothesis that omega 1/ omega 2 agonists, such as zolpidem, increase the frequency of openings in the chloride ionophore, with GABA(A) receptor-dependent and -independent mechanisms. ${ }^{45}$ Subunit modulation of the GABA receptor chloride channel macromolecular complex is hypothesized to be responsible for sedative, anticonvulsant, anxiolytic and myorelaxatant properties. ${ }^{46}$ We suggest involvement of ET and electrostatic properties of the drug.

How can ET influence events in the CNS? A moving electron possessing a negative charge generates an electrical field which can interact with mobile polar species in the CNS. For example, cations are attracted and anions are repelled, entailing participation of electrostatic forces.

\section{Receptors}

The drug acts at the GABA(A) receptor BZ site. Receptor site affinities are shown in Table 1 . The sedative and anticonvulsant activities are due to its action on the alpha-1-GABA(A) receptors. Structural features for high-affinity binding are provided in Table 2 .

\section{Cell Signaling}

Investigations were carried out on signal transduction involving the zolpidem GABA binding site. Throughout most of the developing brain, GABAergic synapses are the first to become functional. ${ }^{48}$ Several features of accompanying signaling change during development, suggesting that signaling in the immature brain may play important roles in the growth of young neurons and establishment of networks. Synaptic currents were examined. GABAergic signaling to newborn neurons occurs in dentate gyrus. ${ }^{49}$ Synaptic currents in mature granule cells were more sensitive to $G A B A(A)$ receptor modulator zolpidem than in newborn cells.

\section{Metabolites}

A number of investigations are available dealing with metabolism of zolpidem. These mainly involve oxidative pathways catalyzed by cytochrome-type enzymes. ${ }^{50-53}$ The products are primarily carboxylic acids generated via alcohol intermediates. Metabolites display poor penetration into the brain and no significant hypnotic activity. ${ }^{53}$ These results provide support for the claim that the parent itself is the active agent.

\section{ROS and OS}

As pointed out in the Introduction, there is extensive evidence for participation of ET functionalities in the therapeutic action of

\section{Table 1 Receptor site and affinity}

\begin{tabular}{lcc}
\hline Receptor site & Affinity & Ref. \\
GABA(A) & Various & 47 \\
Alpha-1 & High & 47 \\
Alpha-2 & Intermediate & 47 \\
Alpha-3 & Intermediate & 47 \\
Alpha-5 & None & \\
Benzodiazepine Type 1 & High & 42 \\
\hline
\end{tabular}

\section{Table 2 Receptor binding and SAR involving amino acid (AA) residues}

\begin{tabular}{lcc} 
AA residues & Binding effect & Ref. \\
Met & Necessary & 26 \\
Phe & Necessary & 26 \\
Gamma-2-subunit loop F & Required for tight docking & 26 \\
(7AA stretch) & \\
Glu, Thr, Arg & Important for high affinity binding & 26 \\
Arg & Hydrogen bonding & 25 \\
\hline
\end{tabular}

drugs, as well as in toxicity. The adverse response is often a result of ROS generation, accompanied by OS, as a consequence of redox cycling entailing ET. However, there are not substantial findings for ROS formation from zolpidem. Toxicity of the drug is quite $l_{0}{ }^{54}$ in contrast with higher levels for many other drugs, which appear to be related to ROS. ${ }^{6-15}$ Another relevant feature is causation of little GSH depletion by the drug, a sign of minor OS. ${ }^{55}$ Reports for other drugs exist in which ET appears to occur, but in the absence of appreciable formation of ROS. For example, amsacrine, a 9-anilinoacridine derivative and anticancer agent, appears to exert its cytotoxic action by poisoning of cellular topoisomerase enzymes. ${ }^{55}$ Evidence indicates that the drug may act as an electron donor in ET reactions, perhaps involving DNA.

Other citations are 5,6-dimethylxanthenone-4-acetic acid (DMXAA) and flavone-8-acetic acid (FAA) which appear to be capable of ET as pyrylium-type species. ${ }^{56}$ There is little indication of ROS and OS. The anticancer agent DMXAA exhibits tumor antivascular activity. A cell-signaling mechanism was proposed involving cytokine inducers that cause tumor necrosis. ${ }^{57}$ Administration of DMXAA or FAA results in the synthesis of TNF, in addition to various cytokines, chemokines and transcription factor.

\section{Side Effects}

One of the common adverse responses to zolpidem use is hallucinations. ${ }^{58-61}$ A proposed mechanism entails changes in the GABAA receptor. ${ }^{58}$ Another suggestion involves a pharmacodynamic interaction between serotonin reuptake inhibition and the drug. ${ }^{59} \mathrm{~A}$ novel concept of hallucination is based on natural bioluminescence and redox processes, in which there is a connection between the neuronal and weak photon emission processes. ${ }^{62}$ Synchronized electrical (redox) signals of neurons can be converted to photon signals by bioluminescent radical ROS and RNS involving signaling pathways. 


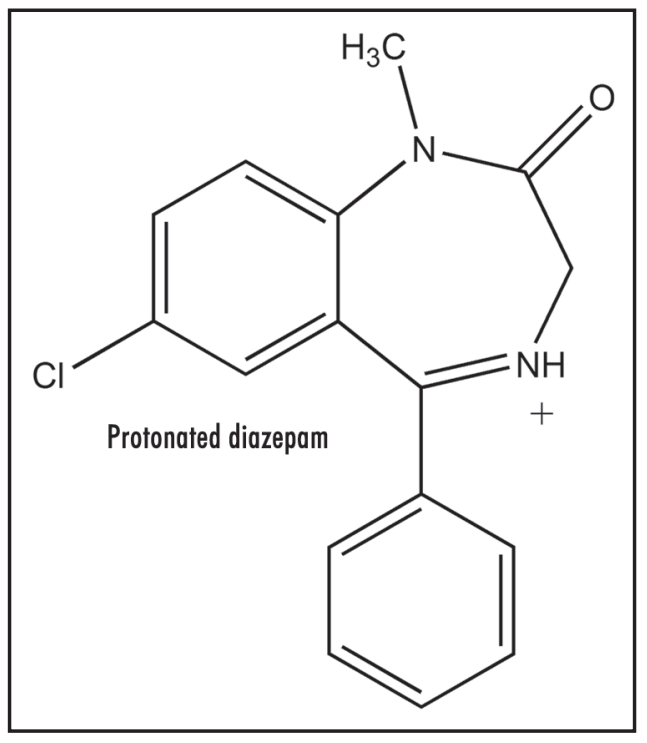

Figure 6. Protonated diazepam.

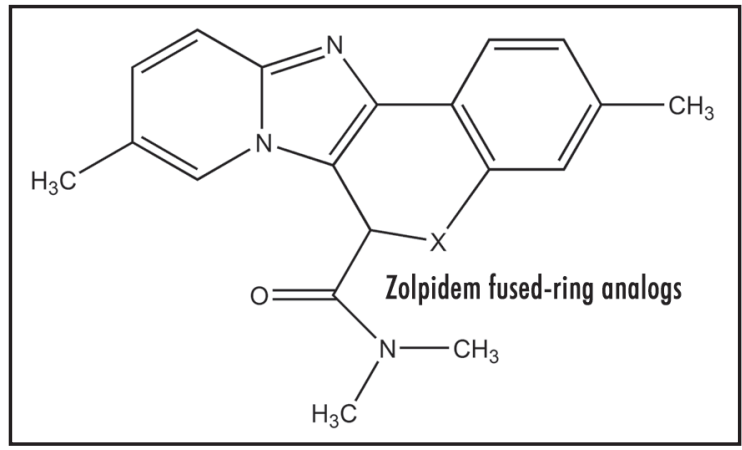

Figure 7. Zolpidem fused-ring analogs.

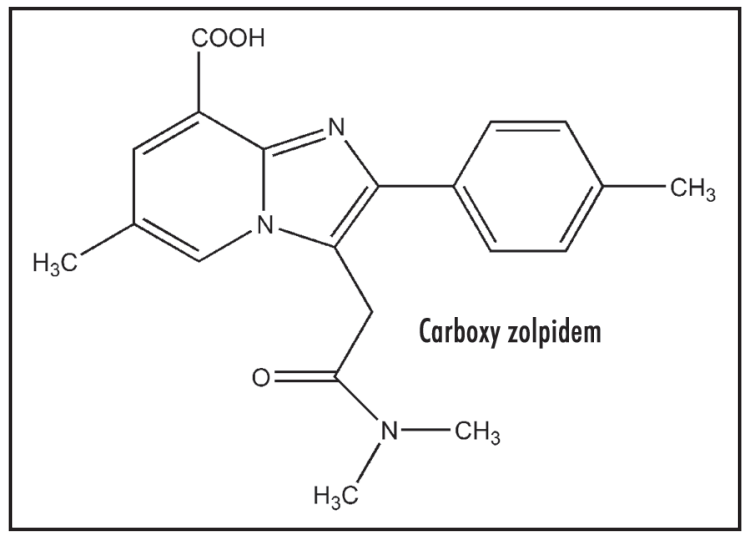

Figure 8. Carboxy zolpidem.

An article dealing with clinical reports cites eight cases of zolpidem abusers who craved not its sedative properties, but rather the anxiolytic and stimulating actions. ${ }^{63}$ It is possible that, for the high doses used, the drug abandons its selectivity for BZ1 receptors and demonstrates all the actions of classic BZs. A review addresses the mode of action for sedative abuse based on the unifying framework of ET, radical involvement and electrochemistry. ${ }^{13}$

\section{Other Sedatives: SAR}

In addition to zolpidem, other prominent sedatives include BZs and barbiturates. Inspection of the literature reveals unifying structural and mechanistic threads. In the case of BZ, there is structural similarity to zolpidem, both being highly conjugated imines, although, interestingly, literature reports usually emphasize differences. By focus on the protonated form of BZ (diazepam) (Fig. 6), the presence of two iminium structures is evident. Thus, both Figures 3 and 6, are members of the important conjugated iminium ET category noted in the Introduction. The BZ drugs influence the activity of all major areas in the CNS, which results in various electrical phenomena. ${ }^{11,64}$ Changes are induced in the electrically excitable properties of spinal neurons. Reduction potentials of various protonated members fall within the physiologically active range. Hence, ET reactions might be a contributing factor. Correlations exist involving reduction potential, structure and drug activity. According to theoretical calculations, electronic effects and ET play important roles in the biochemical behavior.

In relation to barbiturates, although structurally similarity is not close, there is unity in relation to generation of an ET precursor from phenobarbital, namely a catechol. ${ }^{7}$ This class blocks ion channels, and affects membrane potentials and synaptic neurotransmission. Metabolic studies reveal aromatic hydroxylation as an important pathway leading to phenols and a catechol. The catechol group undergoes redox cycling with the corresponding o-quinone involving ET.

\section{Clinical Aspects}

Studies involving clinical pharmacology deal with sedative, anticonvulsant, anxiolytic and myorelaxant drug properties. ${ }^{65}$ Receptor interaction is addressed in an earlier section, as well as some side effects. Clinical studies on other side effects were performed in relation to decrease in performance, rebound effects, memory impairment, anaphylactic reaction, depression and dizziness. The pharmacokinetic profile is also available.

\section{Hypothesis Evaluation}

Experimental determination of the reduction potential of zolpidem and its salt should be made in order to test the hypothesis. It would be interesting to synthesize and test analogs of type (Fig. 7) in which $\mathrm{X}$ is $\mathrm{O}, \mathrm{S}, \mathrm{NH}, \mathrm{CH}_{2}, \mathrm{CR}_{2}, \mathrm{CO}, \mathrm{SO}_{2}$ and $\mathrm{C}=\mathrm{CH}_{2}$. The additional fused ring would exert a favorable influence on ease of electron uptake in ET, due to near coplanarity of the polynuclear system, with resultant increased stabilization of the generated radical anion. On the other hand, these may be an adverse effect on critical features necessary for site binding. Another candidate is (Fig. 8) wherein salt formation results from intramolecular protonation.

\section{Acknowledgements}

Editorial assistance by Thelma Chavez is acknowledged.

\section{References}

1. Kovacic P, Pozos RS. Review: Bioelectronome. Integrated approach to receptor chemistry, radical, electron transfer, electrochemistry, cell signaling and physiological effects based on electron transfer. J Recept Signal Transduct 2007; 27:261-94.

2. Kovacic P, Pozos RS. Cell signaling (mechanism and reproductive toxicity): Redox chains, radicals, electrons, relays, conduit, electrochemistry and other medical implications. Birth Defects Res Part C 2006; 78:333-44.

3. Kovacic P, Becvar LE. Mode of action of anti-infective agents: Emphasis on oxidative stress and electron transfer. Curr Pharmaceut Des 2000; 6:143-67. 
4. Kovacic P, Osuna JA. Mechanism of anticancer agents: Emphasis on oxidative stress and electron transfer. Curr Pharmaceut Des 2000; 6:277-309.

5. Kovacic P, Jacintho JD. Mechanism of carcinogenesis: focus on oxidative stress and electron transfer. Curr Med Chem 2001; 8:773-96.

6. Kovacic P, Jacintho JD. Reproductive toxins: Pervasive theme of oxidative stress and electron transfer. Curr Med Chem 2001; 8:863-92.

7. Kovacic P, Somanathan R. Mechanism of teratogenesis: Electron transfer, reactive oxygen species and antioxidants. Birth Defects Res Part C 2006; 78:308-25.

8. Kovacic P, Sacman A, Wu-Weis M. Nephrotoxins: Widespread role of oxidative stress and electron transfer. Curr Med Chem 2002; 9:823-47.

9. Poli G, Cheesman KH, Dianzani MU, Slater TF. Free radicals in the pathogenesis of liver injury. Pergamon, New York 1989; 1-330.

10. Kovacic P, Thurn LA. Cardiovascular toxins from the perspective of oxidative stress and electron transfer. Curr Vasc Pharmacol 2005; 3:107-17.

11. Kovacic P, Somanathan R. Neurotoxicity: The broad framework of electron transfer, oxidative stress and protection by antioxidants. Curr Med Chem-CNS Agents 2005; 5:249-58.

12. Kovacic P, Pozos RS, Somanathan R, Shangari R, O’Brien PJ. Mechanism of mitochondrial uncouplers, inhibitors and toxins: Focus on electron transfer, free radicals and structureactivity relationship. Curr Med Chem 2005; 5:2601-23.

13. Kovacic P, Cooksy AL. Unifying mechanism for toxicity and addiction by abused drugs: Electron transfer and reactive oxygen species. Med Hypotheses 2005; 64:357-66.

14. Halliwell B, Gutteridge JMC. Free Radicals in Biology and Medicine. New York: Oxford; 1999; 1-859.

15. Kovacic P, Somanathan R. Ototoxicity and noise trauma: Elctron transfer, reactive oxygen species, cell signaling, electrical effects and protection by antioxidants: practical medical aspects. Med Hypotheses 2008; 70:914-23.

16. Kovacic P. Bioelectrostatics: Review of widespread importance in biochemistry. J Electrostat 2007; 66:124-9.

17. Kovacic P, Pozos RS, Draskovich CD. Unifying electrostatic mechanism for receptor-ligand activity. J Recept Signal Transtucd 2007; 27:411-31.

18. Kovacic P, Draskovich CD, Pozos RS. Unifying electrostatic mechanism for phosphates and sulfates in cell signaling. J Recept Signal Transduct 2007; 27:433-43.

19. Kovacic P. Unifying electrostatic mechanism for metal cations in receptors and cell signaling. J Recept Signal Tranduct 2008; 28:153-62.

20. Kovacic P, Wakelin LPG. DNA molecular electrostatic potential: Novel perspectives for the mechanism of action of anticancer drugs involving electron transfer and oxidative stress. Anti-Canc Drug Des 2001; 16:1-10.

21. Oszczapowicz J, Jaroszewska-Manaj J, Golimowska K. Amidines, part 40. Amidinedeuteriochloroform complexes. Influence of amidine basicity on the frequency of C-D stretching vibrations. J Chem Soc Perkin Trans 2 2000; 2343-6.

22. Nyberg K. Alkylaromatic Compounds: In Encyclopedia of Electrochemistry of the Elements, Bard AJ, ed. Marcel Dekker, New York, Vol XI 1978; 45-7.

23. Polievkt MK, Sheinkma AK, Morozova LN. Reactions of cyclammonium cations 22 Influence of substituents at heteroatom on polarographic reduction of pyridinium cations. Khim Geterotsiklich Soedin 1973; 1068-72.

24. Gringauz A. Introduction to Medicial Chemistry, Wiley-VCH, New York 1997; 652.

25. Chen Y-C. The molecular dynamic simulation of zolpidem interaction with gamma aminobutyric acid type A receptor. J Chin Chem Soc (Taiwan) 2007; 54:653-8.

26. Sancar F, Ericksen SS, Kucken AM, Teissére JA, Czajkowski C. Structural determinants for high-affinity zolpidem binding to GABA-A receptors. Mol Pharmacol 2007; 71:38-46.

27. Smith JD, Saykally RJJ. Geissler PL. The effects of dissolved halide anions on hydrogen bonding in liquid water. J Am Chem Soc 2007; 129:13847-56.

28. van Lier H, Drinkenburg WHIM, van Eeten YJW, Coenen AML. Effects of diazepam and zolpidem on EEG beta frequencies are behavior-specific in rats. Neuropharmacology 2004 47:163-74.

29. Vesser SAG, Wolters FLC, Van Der Graaf PH, Peletier LA, Danhof M. Dose-dependent EEG effects of zolpidem provide evidence for GABAA receptor subtype selectivity in vivo. J Pharmacol Expt Therapeut 2003; 304:1251-7.

30. Kopp C, Rudolph U, Tobler I. Sleep EEG changes after zolpidem in mice. Neuroreport 2004; 15:2299-302.

31. Landolt HP, Finelli LA, Roth C, Buck A, Achermann P, Borbély AA. Zolpidem and sleep deprivation: different effect on EEG spectra. J Sleep Res 2000; 9:175-83.

32. Yoshimoto M, Higuchi H, Kamata M, Yoshida K, Shimizu T, Hishikawa Y. The effects of benzodiazepine (triazolam), cyclopyrrolone (zopiclone) and imidazopyridine (zolpidem) hypnotics on the frequency of hippocampal theta activity and sleep structure in rats. Eur Neuropsychopharmacol 1999; 9:29-35.

33. Chen L, Xie J-X, Fung K-S, Yung W-H. Zolpidem modulates GABAA receptor function in subthalmic nucleus. Neurosci Res (Netherlands) 2007; 58:77-85.

34. Kang-Park MH, Wilson WA, Moore SD. Differential actions of diazepam and zolpidem in basolateral and central amygdale nuclei. Neuropharmacology 2004; 46:1-9.

35. Bosman LW, Rosahl TW, Brussaard AB. Neonatal development of the rat visual cortex: synaptic function of GABAA receptor alpha subunits. J Physiol 2002; 545:169-81.

36. Taketo M, Yoshioka T. Developmental change of GABAA(A) receptor-mediated current in rat hippocampus. Neuroscience 2000; 96:507-14.

37. Dunning DD, Hoover CL, Soltesz I, Smith MA, O'Dowd DKJ. GABAA(A) receptormediated miniature postsynaptic currents and alpha subunit expression in developing cortical neurons. Neurophysiol 1999; 82:3286-97.
38. Nett ST, Jorge-Rivera JC, Myers M, Clark AS, Henderson LP. Properties and sex-specific differences of GABAA receptors in neurons expressing gammal subunit mRNA in the preoptic area of the rat. J Neurophysiol 1999; 81:192-203.

39. Defazi RA, Hablitz J. Reduction of zolpidem sensitivity lesion model of neocortical dysgenesis. J Neurophysiol 1999; 81:404-7.

40. Criswell HE, McCown TJ, Moy SS. Oxford GS, Mueller RA, Morrow AL, Breese GR. Action of zolpidem on responses to GABA in relation to mRNA for GABA(A) receptor alpha subunits within single cells: evidence for multiple functional GABA(A) isoreceptors on individual neurons. Neuropharmacology 1997; 36:1641-52.

41. Hollrigel GS, Soltesz I. Slow kinetics of miniature IPSCs during early postnatal development in granule cells of the dentate gyrus. J Neurosci 1997; 17:5119-28.

42. Mereu G, Carcangiu G, Concas A, Passino N, Biggio G. Reduction of reticulate neuronal activity by zolpidem and alpidem, two imidazopyridines with high affinity for type I benzodiazepine receptors. Eur J Pharmacol 1990; 179:339-45.

43. Yang X, Knapp DJ, Criswell HE, Breese GR. Action of ethanol and zolpidem on gammaaminobutyric acid responses from cerebellar Purkinje neurons; relationship to beta-adrenergic receptor input. Alcohol Clin Exp Res 1998; 22:1655-61.

44. Scatton B, Claustre Y, Dennis T, Nishikawa T. Zolpidem, a novel nonbenzodiazepine hypnotic II. Effects on cerebellar cyclic GMP levels and cerebral monoamines. J Pharmacol Exp Ther 1986; 237:659-65

45. Lloyd GK, Danielou G. Thuret F. The activity of zolpidem and other hypnotics within the gamma-aminobutyric acid (GABAA) receptor supramolecular complex, as determined by $35 \mathrm{~S}$-t-butylbicyclophosphorothionate (35S-TBPS) binding to rat cerebral cortex membranes. J Pharmacol Exp Ther 1990; 255:690-6.

46. Ambien CR, from Wikipedia, the free encyclopedia http://en.wikipedia.org/wiki/Ambien CR 2007.

47. Crestani F, Martin JR, Möhler H, Rudolph U. Mechanism of action of the hypnotic zolpidem in vivo. Br J Pharmacol 2000; 131:1251-4.

48. Karten YJG, Jones MA, Jeurling SI, Cameron HA. GABAergic signaling in young granule cells in the adult rat and mouse dentate gyrus. Hippocampus 2006; 16:312-20.

49. Wadiche LO, Bromberg DA, Bensen AL, Westbrook GL. GABAergic signaling to newborn neurons in dentate gyrus. J Neurophysiology 2005; 94:4528-32.

50. Chouinard G, Lefko-Singh K, Teboul E. Metabolism of anxiolytics and hypnotics: benzodiazepines, buspirone, zopilcone and zolpidem. Cell Mol Neurobiol 1999; 19:533-52.

51. Pichard L, Gillet G, Bonfils C, Domergue J, Thénot JP, Maurel P. Oxidative metabolism of zolpidem by human liver cytochrome P450S. Drug Metab Dispos 1995; 23:1253-62.

52. Lewis JH, Vine JH. A simple and rapid method for the identification of zolpidem carboxylic acid in urine. J Anal Toxicol 2007; 31:195-9.

53. Garrigou-Gadenne D, Burke JT, Durand A, Depoortere H, Thénot JP, Morselli PL. Pharmokinetics, brain distribution and pharmaco-electrocorticographic profile of zolpidem, a new hypnotic, in the rat. J Pharmacol Exp Ther 1989; 248:1283-8.

54. Berson A, Descatoire V, Sutton A, Fau D, Maulny B, Vadrot N, Feldmann G, Berthon B, Tordjmann T, Pessayre D. Toxicity of alpidem, a peripheral benzodiazepine receptor ligand, but not zolpidem, in rat hepetocytes: Role of mitochondrial permeability transition and metabolic activation. J Pharmacol Exp Therp 2001; 299:793-800.

55. Baguley BC, Wakelin LPG, Jacintho JD, Kovacic P. Mechanisms of action of DNA intercalating acridine-based drugs: How important are contributions from electron transfer and oxidative stress? Curr Med Chem 2003; 24:2643-58.

56. Kovacic P. Fundamental, electron transfer mechanism by pyrylium-type ions for the anticancer drugs 5,6-dimethylxanthenone-4-acetic acid (DMXAA) and flavone-8-acetic acid. Curr Med Chem-Anti-cancer Agents 2005; 5:501-6.

57. Wan LC, Reddy CB, Baguley BC, Sutherland R, Ching L-M. Induction of tumor necrosis factor and interferon-gamma in cultured murine splenocytes by the antivascular agent DMXAA and its metabolites. Biochem Pharmacol 2004; 67:937-45.

58. Tsai MJ, Huang YB, Wu PC. A novel clinical pattern of visual hallucination after zolpidem use. J Toxicol Clin Toxicol 2003; 41:869-72.

59. Elko CJ, Burgess JL, Robertson WO. Zolpidem-associated hallucination and serotonin reuptake inhibition: A possible interaction. J Toxicol Clin Toxicol 1998; 36:195-203.

60. Toner LC, Tsambiras BM, Catalano G, Catalano MC, Cooper DS. Centrakl nervous system side effects associated with zolpidem treatment. Clin Neuropharmacol 2000; 23:54-8.

61. Coleman DE, Ota K. Hallucinations with zolpidem and fluoxetine in an impaired driver. J Forensic Sci 2004; 49:392-3.

62. Bokkon I, D'Angiulli A. Emergence and transmission of visual awareness through optical coding in the brain: A redox molecular hypothesis on visual mental imagery. Biosci Hypoth 2009; In press.

63. Liappas IA, Malitas PN, Dimopoulos NP, Gitsa OE, Liappas AI, Nikolaou Ch K, Christodoulou GN. Zolpidem dependence case series: Possible neurobiological mechanisms and clinical management. J Psychopharmacol 2003; 17:131-5.

64. Crawford PW, Kovacic P, Gilman NW, Ryan MD. Charge transfer mechanism for benzodiazepine (BZ) action. Correlation of reduction potential of BZ iminium with structure and drug activity. Bioelectochem Bioenerg 1986; 16:407-26.

65. Engel K. Physicians Desk Reference, Thomson Health Care, Montvale NJ 2008 62:2799-801. 


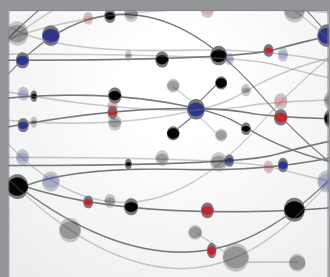

The Scientific World Journal
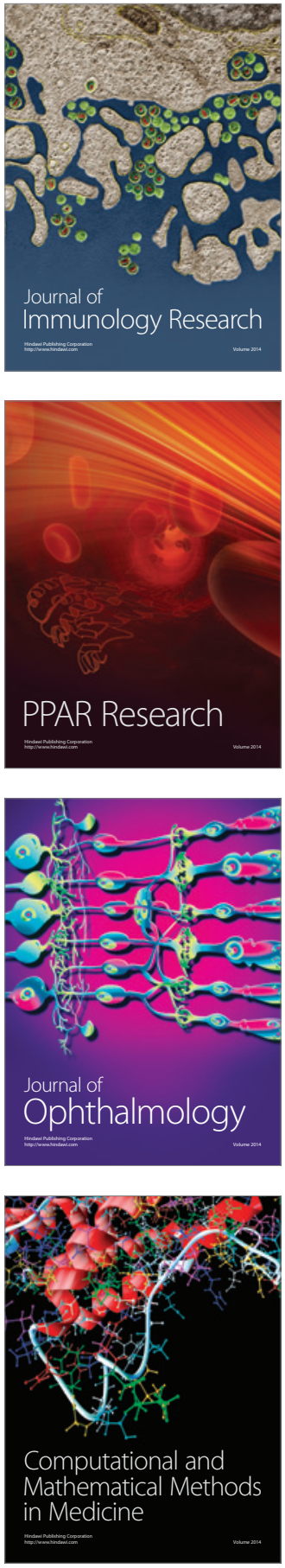

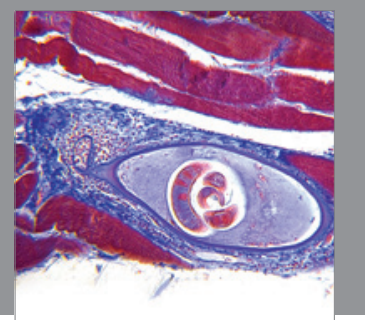

Gastroenterology

Research and Practice
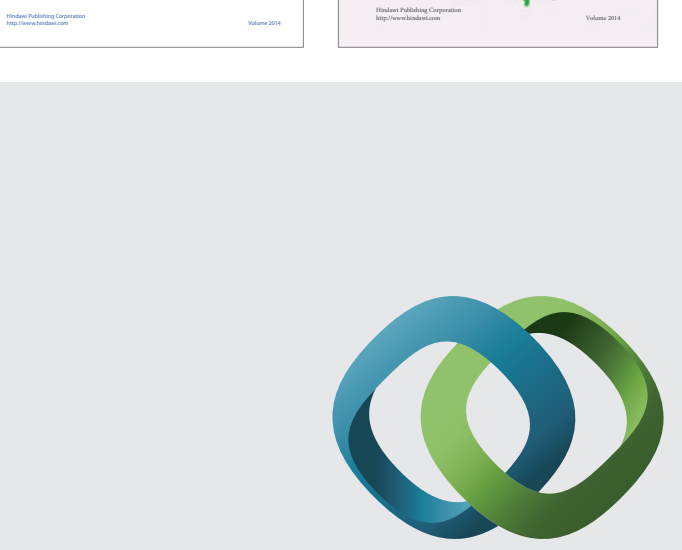

\section{Hindawi}

Submit your manuscripts at

http://www.hindawi.com
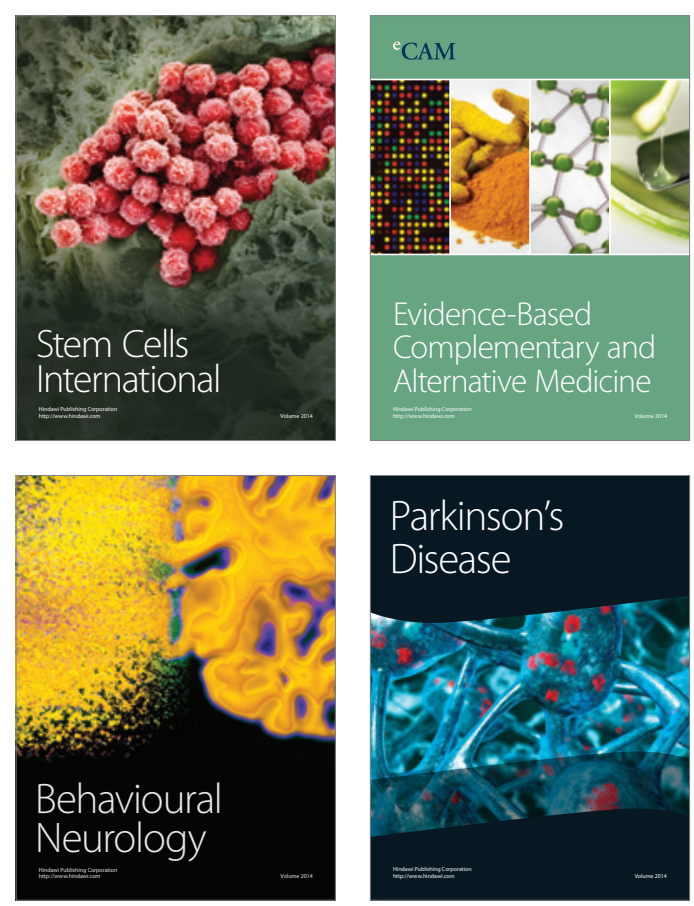

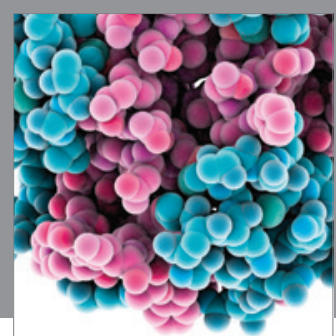

Journal of
Diabetes Research

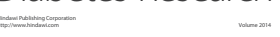

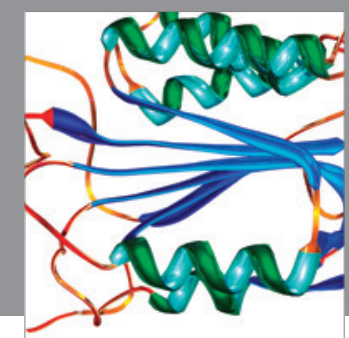

Disease Markers
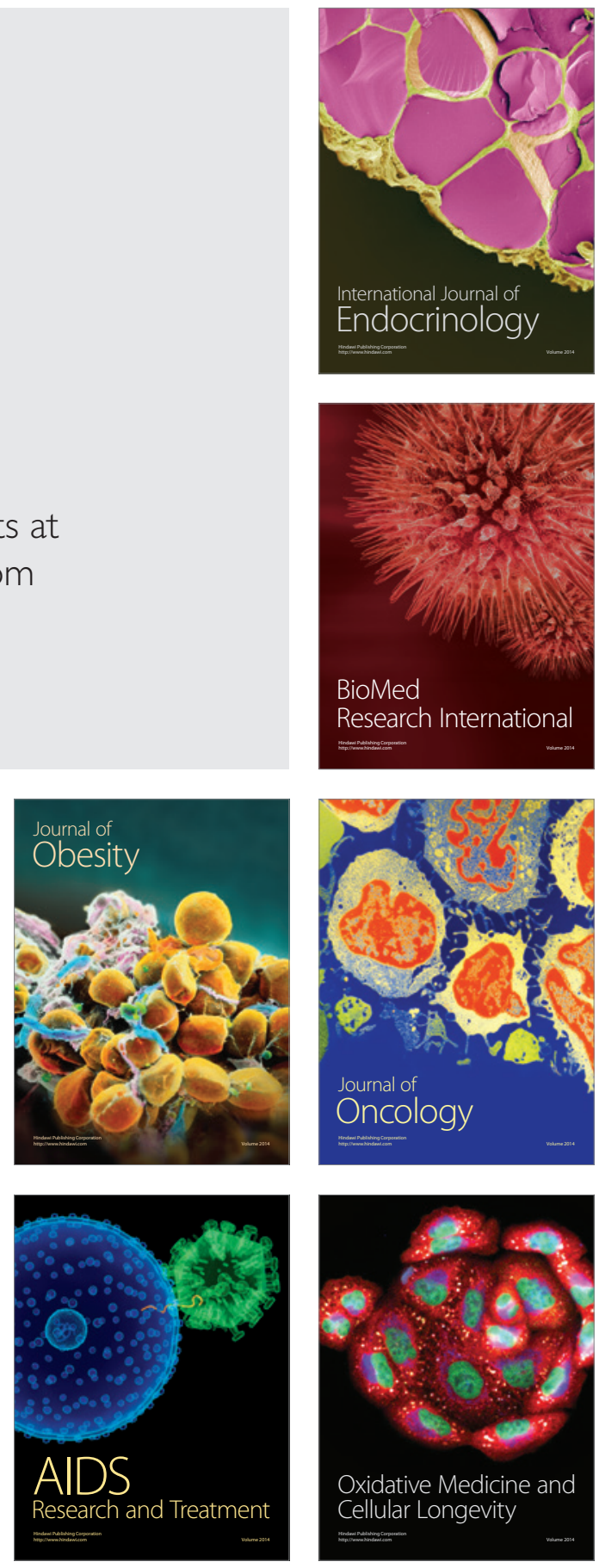